
Military Technical College, Kobry El-Kobbah, Cairo, Egypt

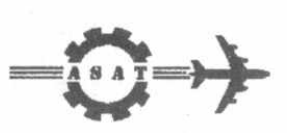

$9^{\text {th }}$ International Conference On Aerospace Sciences \& Aviation Technology

\title{
MULTILEVEL OPTIMIZATION OF LAMINATED COMPOSITE STRUCTURES
}

\author{
NASR ${ }^{1}$ A.N. AND NEGM ${ }^{2}$ H.M.
}

\section{ABSTRACT}

This paper presents a multilevel approach to the optimal design of laminated composites with consideration of natural frequency constraints. The Ply thicknesses and orientations are designed to minimize the volumes of plates subjected to constraints on the first natural frequency. The optimization process is carried out in a dual scheme. In the first optimization level the optimal ply orientations which give the maximum natural frequency are determined. In the second level the optimal ply thicknesses are determined so as to minimize the structural weight, while maintaining the ply angles obtained in the first level. The process is repeated several times until convergence is obtained. Weight minimization is performed by two different methods: the constrained gradient travel method and the optimality criterion method.

Key Words: Optimum, Laminated Composite Plate, Dynamic and Static.

\section{NOMENCLATURE}

$\begin{array}{ll}\ominus & \text { Ply angle } \\ \{\Phi\} & \text { Vector of ply orientations } \\ \omega_{f} & \text { Fundamental natural frequency. } \\ {[K]} & \text { Global stiffness matrix of the structure. } \\ {[M]} & \text { Global mass matrix of the structure. } \\ \{U\} & \text { Vector of mode shape } \\ L & \text { Number of plies in a given design. } \\ \{t\} & \text { Vector of Ply thickness. }\end{array}$

\section{INTRODUCTION}

In 1960 Schmit proposed coupling of the Finite Element Analysis with Mathematical Programming methods for structural design optimization purposes Schmit [5].

1General Manger A.O.I. Aircraft Engine Factory, Helwan, Cairo, Egypt

2Professor of Aircraft Structures, Aerospace Engineering Department, Cairo University 
In this approach structural design is treated as a problem of mathematical extremization of an objective function in an ' $n$ ' dimensional design variable space constrained by behavioral constraints on stresses, displacements and frequencies. The search for the extremum is carried out by methods of linear and non-linear Programming techniques. The mathematical programming (numerical search) techniques may be grouped into two general categories. One is the conversion of a constrained design problem into a sequence of unconstrained problems where constraints on the design are treated indirectly as penalty functions. The other approach consists of treating the constraint functions directly during the design process (direct method). Exampies of the mathematical programming methods are the gradient projection, steepest descent and feasible direction methods. In the method of feasible direction Vanderplaats [8], the design is changed so that the trajectory in the design space follows the constraint surfaces.along directions that decrease the objective function as quickly as possible but never leaves the feasible domain. A non-linear constraint cannot be followed continuously because the algorithm must take finite linear steps. Therefore a search vector must be determined which both decreases the objective function and does not violate the constraints for a finite move.

In 1971 Venkayya [9] presented the Optimality Criteria approach. In the past twenty years several optimality criteria were derived for a number of structural design applications. The well-known computer programs OPTSTAT, OPCOMP, OPTIM, ASOS, FASTOP, DESAP, etc use discrete optimality criteria approaches as a basis for structural optimization. All these programs are based on the use of the finite element method as an analysis tool. They can be used to optimize isotropic, anisotropic and layered composite structures.

In Ref. [6] a method is presented for minimum weight design of symmetric fibercomposite laminates subject to multiple in-plane loading conditions, which takes into account membrane stiffness requirements and strength limitations. The problem is treated as a non-linear mathematical programming problem in which the thicknesses of laminae placed at preassigned orientation angles are treated as the only design variables. The non-linear programming formulation is transformed into a sequence of linear programming problems employing an adaptation of the method of inscribed hyperspheres in which only critical and near critical constraints are considered at each stage in the procedure. The resulting sequence of linear programming problems can be easily and quickly solved by using a standard Simplex algorithm.

Fleury [2] presents a new and powerful mathematical programming method in which optimization is performed using a new dual method employing mixed variables. The method employs mixed direct/reciprocal design variables in order to get conservative, first-order approximations to the objective function and constraints (convex linearization). By this approach the primary optimization problem is replaced by a sequence of explicit subproblems, each subproblem being convex and separable, and is efficiently solved by using a dual formulation. The dual problem 
consists in maximizing an auxiliary function that depends only on the Lagrangian multipliers associated with the main primal constraints. Thus the effective dimensionality of the dual problem never exceeds the number of active behavioral constraints. The application of this method to aerospace structures with a large number of constraints has demonstrated its power and generality.

Sobieszczanski-Sobieski [7] presented a development of a general multilevel optimization method in which the structure is classified into three substructural levels. This method is suitable for realistic structures having many components and carrying a large number of loading cases and hindered by an excessive number of design variables and constraints. For example, a portal framework can be decomposed into individual beams, each beam is a box that can be further decomposed into stiffened plates. By this way large optimization problems can be broken into smaller subproblems, and a coordination scheme is formulated to preserve the coupling among these subproblems.

Optimization methods based on an optimality criterion to design a minimum weight structure are known as the indirect methods. This is because in these methods the objective is to obtain a design that satisfies a specified criterion, and by doing so we indirectly minimize the weight of the structure. Developing an efficient algorithm based on such a criterion which effectively handles all types of constraints would be impractical and generally unnecessary. In most structures it is likely that one can predict the type of constraint which will be active at the optimum, and use the algorithm based on that constraint. The optimality criterion derived for all the constraints imposed on the structure is equivalent to the Kuhn-Tucker conditions of nonlinear mathematical programming Kiusalaas [3].

Structural optimization methods are iterative by their nature, and hence optimization problems cannot be solved in one step. In the optimality criteria methods, the optimization procedure during each iteration cycle can be divided into two phases. First the structure is analyzed in order to determine its behavior under the applied loads. Then, the material is redistributed, modifying the design variables so that the total weight is minimized and the design satisfies the applicable optimality conditions. To carry out this last step the optimality criterion is used to derive a recurrence relation which is used for updating the design variables.

\section{MULTILEVEL APPROACH}

\subsection{Structural Optimization with Frequency Constraints}

This work concerns structural optimization with dynamic frequency constraints because of its importance in structural design. It is not uncommon that structural components of flight vehicles and automobiles fail due to fatigue fracture as a result of excessive vibrations. In most low-frequency vibration problems the response of 
the structure to dynamic excitation is primarily a function of its fundamental frequency and mode shape .In such cases, the ability to manipulate the fundamental frequency can significantly improve the performance of the structure. In aircraft design, frequency requirements are often imposed on control surfaces and major structural components as flutter prevention measures.

\subsection{Optimization Procedure}

An analytical procedure is presented for the determination of the least weight design of a laminated composite structure, which satisfies a specific frequency requirement. The design variables are the ply angles and ply thicknesses $\left(\theta^{1}, t^{1}, \theta^{2}, t^{2}, \ldots . \theta^{\mathrm{L}}, \mathrm{t}^{\mathrm{L}}\right)$, where $L$ is the numbers of plies in the given design.

The approach is to modify an existing structure by:

- First, ranking its plies to have the best stacking sequence which gives the highest natural frequency, and using this modified design as a starting design .

- Second, maximizing the natural frequency of the structure by changing ply angles in steps to have a structure with the maximum possible natural frequency. This is accomplished by using gradient equations, which express the rate of change of frequency with respect to changes of ply angles. The gradients can be evaluated in terms of ply stiffness and mass matrices and the associated mode shape.

- Third, minimizing structural weight by minimizing ply thicknesses while maintaining ply angles, which were determined in the previous level of frequency maximization. Minimization of ply thicknesses is continued until the structural frequency is reduced to the minimum acceptable limit, i.e the frequency gain achieved in the first level is consumed.

- Fourth, switching again to the frequency maximization level trying to achieve any further frequency gain, and if there is still any, we switch to weight minimization and continue until no more weight decrease is possible.

Thus the optimization process is carried out in a dual scheme where ply orientations and thicknesses are designed separately at two independent levels of optimization. An example is given to demonstrate the applicability and effectiveness of the proposed method.

In the present work the weight minimization is accomplished by using two different methods: the optimality criterion method and the constrained gradient travel method. The equations governing the redesign process in both levels of optimization (frequency maximization and weight minimization) are based on gradient equations similar to the gradient equations which were first published by Zarghamee [10]. 


\subsection{Finite Element Analysis And Gradient Equations}

\subsubsection{Sensitivity Analysis}

Design sensitivity analysis plays a central role in structural optimization, as virtually all optimization methods require the computation of derivatives of a structural response quantity with respect to design variables. Thus the design derivative (design sensitivity) analysis portion of any structural optimization algorithm constitutes a major segment of the total calculations. Sensitivity derivatives are used for studying the effect of parametric modifications and calculating the search directions for finding an optimum design.

In the present finite element model of a composite plate, a $C^{\circ}$ finite element developed by the author in reference Nasr [1] is adopted for the structural and free vibration analyses. The element as mentioned there has five degrees-of-freedom (three displacements and two shear rotations) per node. The equation of motion of a structure modeled with discrete elements and assembled by the finite element technique is expressed in matrix form as:

$$
[M]\{\ddot{i}\}+[K]\{u\}=\{F\}
$$

For static analysis Eq. (1) reduces to

$$
[K]\{u\}=\{F\}
$$

For free-vibration analysis, Eq.( 1) becomes an eigen value problem:

$$
[\mathrm{K}]\{\mathrm{u}\}-\omega^{2}[\mathrm{M}]\{\mathrm{u}\}=0
$$

in which $[K]$ and $[M]$ are the global stiffness and mass matrices, respectively, $\{\mathrm{U}\}$ is the mode shape vector, and $\omega^{2}$ is the square of the corresponding natural frequency.

\subsubsection{Gradient Equations for Frequency Maximization}

If Eq. (3) is differentiated with respect to ply angles $\left(\theta_{\mathrm{j}}\right)$ we get :

$$
[\mathrm{K}]\left\{\mathrm{u}_{j}\right\}-\omega^{2}\left[\mathrm{M}\left\{\mathrm{u}_{, \theta_{j}}\right\}=\omega_{, \theta_{j}}^{2}[\mathrm{M}]\{\mathrm{u}\}-\left[\mathrm{K}_{, \theta_{j}}\right]\{\mathrm{u}\}+\omega^{2}\left[\mathrm{M}_{, \theta_{j}}\right]\{\mathrm{u}\}\right.
$$


Premultiplication of this equation by $\{u\}^{\top}$ makes the left hand side equal to zero due to symmetry of $[\mathrm{K}]$ and $[\mathrm{M}]$, and satisfaction of Eq.(3).

The equation therefore becomes

$$
0=\omega^{2}, \theta_{j}\{u\}^{T}[M]\{u\}-\{u\}^{T}\left(\left[K_{, \theta_{j}}\right]\{u\}+\omega^{2}\left[M_{, \theta_{j}}\right]\right)\{u\}
$$

For convenience, the generalized mass is normalized to unity, so the basic gradient equation can be written in the form:

$$
\omega^{2}{ }_{\theta_{j}}=\{u\}^{\mathrm{T}}\left(\left[\mathrm{K}_{\theta_{\theta_{j}}}-\omega^{2} \mathrm{M}_{\theta_{\theta_{j}}}\right]\right)\{\mathrm{u}\}
$$

But since the mass matrix is not a function of ply angles then Eq. (5) can be written as :

$$
G_{\theta_{j}}=\omega^{2}{ }_{, \theta_{j}}=\{\mathrm{u}\}^{\mathrm{T}}\left[\mathrm{K}_{, \theta_{j}}\right]\{\mathrm{u}\}
$$

where $\{u\}$ is the mode shape vector after M-orthonormalization.

\subsubsection{Gradient Equations for Weight Minimization}

If Eq. (3) is differentiated with respect to ply thicknesses $\left(t_{j}\right)$ the result may be written as:

$$
[\mathrm{K}]\left\{\mathrm{u}_{, \mathrm{t}_{\mathrm{j}}}\right\}-\omega^{2}[\mathrm{M}]\left\{\mathrm{u}_{, \mathrm{t}_{\mathrm{j}}}\right\}=\omega_{, \mathrm{t}_{\mathrm{j}}}^{2}[\mathrm{M}]\{\mathrm{u}\}-\left[\mathrm{K}_{, \mathrm{t}_{\mathrm{j}}}\right]\{\mathrm{u}\}+\omega^{2}\left[\mathrm{M}_{, t_{\mathrm{j}}}\right]\{\mathrm{u}\}
$$

Premultiplication of this equation by $\{u\}^{\top}$ makes the left hand side equal to zero due to the symmetry of $[\mathrm{K}]$ and $[\mathrm{M}]$, and the satisfaction of Eq. (3).

The equation therefore becomes

$$
0=\omega_{t_{1}}^{2}\{u\}^{T}[M]\{u\}-\{u\}^{T}\left(\left[K_{, t},\right]\{u\}+\omega^{2}\left[M_{, t_{1}}\right]\right)\{u\}
$$

If the mode shape is orthnormalized, then the basic gradient equation can be written in the form:

$$
\mathrm{G}_{\mathrm{t}_{j}}=\omega^{2}{ }_{, t_{j}}=\{u\}^{\mathrm{T}}\left(\left[\mathrm{K}_{, \mathrm{t}_{j}}\right]-\omega^{2}\left[\mathrm{M}, \mathrm{t}_{\mathrm{j}}\right]\right)\{\mathrm{u}\}
$$




\subsection{Frequency-Maximization Level}

At this level the objective is to find the optimal fiber direction of each layer which gives the maximum natural frequency of the structure.

In mathematical form, the optimization problem is:

$$
\begin{gathered}
\text { Minimize } \\
\varphi(\theta)=-\frac{\omega_{f}^{2}}{\omega_{1}^{2}} \\
\text { Subject to } \\
\theta^{l} \leq \theta \leq 180^{\circ} \\
\text { Where }\left\{\{\theta\}=\left\{\theta_{1}, \theta_{2}, \ldots, \theta_{l}\right\}\right.
\end{gathered}
$$

Where $\varphi$ is the vector of ply orientations.

$\omega_{f}$ is the fundamental natural frequency, and $\omega_{1}$ is the lower boundary of the natural frequency of the first vibration mode.

The redesign process in this phase is as follows:

1. Increase the square of the fundamental frequency of the structure to some specified value in each redesign cycle by changing the ply angles $\left(\theta_{i}\right)$ in proportion to the gradients defined by Eq. (6)

$$
\Delta \theta_{j}=\mathrm{KG}_{\theta_{\mathrm{j}}}
$$

where $\mathrm{K}$ is the step size.

The corresponding increase in the square of the natural frequency is given by:

$$
\Delta \omega^{2}=\sum_{\theta_{j=1}=1}^{\theta_{1}=L} \mathrm{G}_{\theta_{j}} \Delta \theta_{\mathrm{j}}
$$

For a prescribed change in frequency, the step size $(K)$ may be evaluated from:

$$
\mathrm{K}=\frac{\Delta \omega^{2}}{\Sigma \mathrm{G}^{2}}
$$

2. At the end of the redesign cycle the fundamental frequency of the new design is calculated, and the new gradient of each ply $\left(G_{\theta j}\right)$ is calculated. 
3. Repeat steps 1 and 2 till the increment of frequency in one redesign cycle become less than a certain prescribed ratio, e.g. $1 \%$.

\subsection{Weight -Minimization Level}

At this level the fiber directions obtained at the previous level are maintained while the thickness of each ply is reduced so that the total weight of the structure is minimized and the frequency constraint is satisfied. The minimization problem is defined in the following mathematical form:

$$
\begin{aligned}
& \text { Minimize } \\
& W=w(t) \\
& \text { Subject }{ }^{*} \text { to } \\
& \omega_{f} \geq \omega^{*} \\
& t^{\prime} \leq t \leq t^{u}
\end{aligned}
$$

Where $t$ is the vector of layer thicknesses, superscript I and $u$ denote the lower and upper bounds of the design variables.

This minimization problem is treated by two approaches. The first approach is the constrained gradient travel approach, and the second one is the optimality criterion approach.

\subsubsection{Constrained Gradient Travel Approach}

This approach has been presented by Rubin [4] for use with structures whose characteristic matrices (stiffness and mass) are linear functions in their design variables. The approach is developed in the present work for application to complicated structures. The weight of structural members is minimized in proportion to their effectiveness in modifying the fundamental frequency.

The main idea of this work is to make changes in the weight of the composite layer in a way that the effect of these changes on the decrease of the structure fundamental frequency does not exceed the gain $\left(\Delta \omega^{2}\right)$ in the frequency which has been achieved in the frequency maximization level. Hence,

$$
W_{m}^{I+1}-W_{m}^{l}=\eta \frac{\partial \omega^{2}}{\partial W_{m}}
$$

Or

$$
t_{m}^{t+1}-t_{m}^{I}=\eta \frac{G_{m}}{w_{m}^{2}}
$$


Where $\eta$ is a constant of proportionality.

In order not to violate the frequency constraint we must have

$$
\sum_{m=1}^{L} G_{m} \Delta t_{m}=\Delta \omega^{2}
$$

Substituting (16) into (17) we get:

$$
\eta=\frac{\Delta \omega^{2}}{\sum_{m=1}^{L}\left(\frac{G_{m}}{w_{m}}\right)^{2}}
$$

The redesign process in this phase is as follows:

1. Calculate the difference $\left(\Delta \omega^{2}\right)$ between the maximum frequency which has been obtained from the frequency maximization level and the lowest allowable frequency level.

2. Calculate the sum of the squares of layer gradients $\left(G_{t}\right)$.

3. From steps 1,2 calculate the step size of minimizing the thickness according to Eq. (18).

4. The layer thicknesses are minimized according to Eq.(16) in steps.

5. Calculate the fundamental frequency of the new design, and check satisfaction of the frequency constraint. If there is still positive difference between the fundamental frequency and its lower limit, continue minimizing the layer thicknesses according to steps (1-5) till the frequency constraint or any other side constrain becomes critical

In step 3 we found from experience that it is better to minimize the thicknesses such that

$$
0.99 \Delta \omega^{2}=\sum_{m=1}^{L} G_{m} \Delta t_{m}
$$

The factor $(0.99)$ multiplied by the square of the difference in frequencies gives the frequency constraint a safe margin. Without using this factor the frequency constraint sometimes becomes active or slightly violated. 


\subsubsection{Optimality Criterion}

The minimum weight design of structures subjected to frequency constraints can be alternatively handled by the optimality criterion method. An optimality criterion method can be used to solve the optimization problem cited in eqn.(14).

The optimization problem is to minimize the total structural weight

$$
W=\sum_{m=1}^{L} \rho_{m} A_{m} t_{m}=\sum_{m=1}^{L} w_{m} t_{m}
$$

Under the constraints

$$
\begin{aligned}
& \omega_{f} \geq \omega^{*} \\
& t^{u} \geq t_{m} \geq t^{l} \quad m=1,2, \ldots, L
\end{aligned}
$$

The corresponding Kuhn-Tucker conditions ( necessary conditions for $W$ to be a local minimum Kiusalaas [3]) are :

$$
w_{m}-\lambda\left(\omega^{2}\right)_{, m}\left\{\begin{array}{lll}
=0 & \text { if } & t^{l}<t_{m}<t^{u} \\
\geq 0 & \text { if } & t_{m}=t^{l} \\
\leq 0 & \text { if } & t_{m}=t^{u}
\end{array}\right.
$$

and

$$
\lambda\left\{\begin{array}{lll}
>0 & \text { if } & \omega=\omega^{*} \\
=0 & \text { if } & \omega>\omega^{*}
\end{array}\right.
$$

where $\lambda$ are Lagrangian multipliers.

From section 2.3. The frequency gradients are given by:

$$
\left(\omega^{2}\right)_{, m}=\{u\}^{T}\left(\left[K_{, m}\right]-\omega^{2}\left[M_{, m}\right] R u\right\}
$$

Where the eigenvectors are assumed to be orthonormalized. Note that only elements linked to the $\mathrm{m}$-th design variable will contribute to $\left[K_{, m}\right]$ and $\left[M_{, m}\right]$ 


\subsubsection{Weight Minimization Equation}

The proposed design algorithm is equivalent to solving the optimality criterion equation (22) and (23) by successive iteration. Multiplying both sides of $(22)$ by $(1-\alpha)\left(t_{m}\right)$, where $0<\alpha<1$, and rearranging terms we get

$$
t_{m}-s_{m} t_{m}\left\{\begin{array}{lll}
=0 & \text { if } & t^{t}<t_{m}<t^{u} \\
\geq 0 & \text { if } & t_{m}=t^{l} \\
\leq 0 & \text { if } & t_{m}=t^{u}
\end{array}\right.
$$

where

$$
S_{m}=\alpha+\frac{1-\alpha}{w_{m}} \lambda\left(\omega^{2}\right), m
$$

Equation (25) is taken as the recursive design formula:

$$
t_{m}^{I+1}=\left\{\begin{array}{lll}
s_{m} t_{m}^{I} & \text { if } & t^{l}<t_{m}^{I}<t^{u} \\
t^{l} & \text { if } & S_{m} t_{m}^{I}<t^{l} \\
t^{u} & \text { if } & S_{m} t_{m}^{I}>t^{u}
\end{array}\right.
$$

Equation (27) is taken as the recursive design formula:

$$
\begin{aligned}
& t_{m}^{I+1}=\left(\alpha+\frac{(1-\alpha)}{w_{m}} \lambda G_{m}\right) t_{m}^{I} \\
& \text { or } \\
& t_{m}^{I+1}-t_{m}^{I}=(1-\alpha)\left(\lambda \frac{G_{m}}{w_{m}}-1\right) t_{m}^{I}
\end{aligned}
$$

where ${ }^{I+1}$ represent the improved design variables. The parameter $\propto$ is a userspecified relaxation factor that determines the magnitude of the redesign vector. The optimal value of $\propto$ usually lies between 0.5 and 0.85 .

During the redesign process the $m$ layers of the structure are classified into three groups such that group" $p^{\prime}$ contains all the layers for which $t_{m}^{l}<t_{m}^{I+1}<t_{m}^{u}$, group "q" contains all the layers for which $t_{m}^{I+1}<t^{l}$, and group "s" contains all the layers for which $t_{m}^{I+1}>t^{u}$.

In order to change the fundamental frequency of the structure by a certain amount $\Delta \omega 2$, we must have: 
$\Delta \omega^{2}=\left(\omega^{I+1}\right)^{2}-\left(\omega^{I}\right)^{2}=\sum_{m=1}^{L}\left(\omega^{2}\right)_{, m}\left(t_{m}^{I+1}-t_{m}^{I}\right)$

Or

$$
\left.\Delta \omega^{2}=\left(\omega^{*}\right)^{2}-\left(\omega_{1}\right)^{2}=\sum_{p} G_{m}\left(\left(t_{m}\right)_{I+1}-\left(t_{m}\right)_{I}\right)+\sum_{q} G_{m}\left(\left(I^{I}\right)-\left(t_{m}\right)_{I+1}\right)+\sum_{s} G_{m}\left(\left(I^{\mu}\right)-\left(t_{m}\right)_{I+1}\right)\right)
$$

We estimate the frequency change from a linear approximation using equations (29) and (30). When the frequency constraint is active, the non-zero lagrangian multipliers can be determined from the equation $\omega^{l+1}=\omega^{*}$, where $\omega^{I+1}$ is the fundamental frequency of the improved design.

In order to have a feasible design whose frequency is not violating the frequency constraint, the lagrangian multipliers are determined as:

$$
\lambda=\frac{(1-\alpha) \sum_{p} G_{m} t_{m}^{I}-\sum_{q} G_{m}\left(t^{l}-t_{m}^{I}\right)-\sum_{s} G_{m}\left(t^{u}-t_{m}^{l}\right)}{(1-\alpha) \sum_{p} \frac{G_{m}^{2}}{w_{m}} t_{m}^{l}}
$$

The redesign process is as follow:

1. Calculate the frequency gain $\left(\Delta \omega^{2}\right)$, which is the difference between the fundamental frequency in this cycle and the lower allowable limit. If the frequency constraint is not active $(\lambda=0)$, then the redesign eqn.(27)becomes a uniform downward scaling operation $t_{m}^{I+1}=\alpha t_{m}^{i}$. If all the resulting thicknesses of the layers satisfy the side constraint $t^{l}<t^{m}<t^{u}$, then the improved design is feasible and the iteration cycle is completed; otherwise go to the next step.

2. If the frequency constraint is active $(\lambda>0)$, calculate $\lambda$ according to eqn.( 31$)$, then go to next step.

3 For group " $\mathrm{p}$ "(which contains the active layers), determine their new improved design according to Eq.(27). In order to have an improved feasible design, the thiknesses of layers in group "q" will have to be equated to $t^{l}$ and those of group "s" will have to be equated to $t^{4}$.

4. The redesign process is automatically terminated if either the number of iteration cycles exceeds a certain prescribed number or the percentage weight decrease in two successive redesign cycles is less than $1 \%$. 


\section{NUMERICAL EXAMPLE}

Consider the following example problem:

Square plate, simply supported, 4-plies. Side-to-thickness ratio $=20$, 4-layer angle ply $\left(0 \% 45^{\circ} \%-45^{\circ} / 90^{\circ}\right)$.

Material: $\mathrm{E} 1 / \mathrm{E} 2=40, \mathrm{G} 12 / \mathrm{E} 2=0.6, \mathrm{G} 23 / \mathrm{E} 2=0.5, \mathrm{E} 2=0.75 \times 10^{6} \mathrm{PSI}$

$v 12=0.25, \mathrm{G} 13=\mathrm{G} 12, v 13=v 12$ and density $=1.0$

The desired lower limit of eigen value $=900$

Or the desired lower limit of fundamental frequency $=30 \mathrm{rad} . / \mathrm{sec}$

$\mathrm{t}$ (ply thickness) $\geq 0.005$

\section{Laminate Ranking}

In this example laminate ranking has been performed for the given structure before frequency maximization to determine the best starting point (structure with the highest frequency).

The results of ranking are shown in Table 1

\section{Frequency Maximization Level}

The frequency maximization history is plotted in Fig. (1). The history of change of ply orientations is given in Table 2

\section{Weight Minimization Level}

We refer to the constraint gradient travel approach as C.G.T and to the optimality criterion approach as O.C.

The history of weight minimization by the two methods is plotted in Fig. (2) and Fig.( 3).

It is seen that the optimality criterion approach has reached a lower weight design, but using one more iteration cycle. However, in other example problems the opposite occurred.

\section{CONCLUSION}

This paper presents a multilevel approach for redesigning composite plate structures to obtain the least weight configuration, which has its fundamental frequency equal to some specified value. Layer thicknesses and fiber directions are designed 
separately on two levels of optimization. Constrained gradient travel is used at the first level to determine the optimal fiber directions, which maximize the structure's stiffness. In the second level the optimal layer thicknesses which minimize the structure's weight are obtained by two different methods: The constrained gradient method and the optimality criterion method. The effects of the transverse shear deformation on the optimum design of the structures are included. The feasibility of the approaches is illustrated by a numerical example, which shows that the procedures are convergent and capable of determining optimized configurations in a few redesign cycles. Numerical results show that no advantage of one of the two weight minimization approaches over the other.

Table 1 Laminate ranking results for example 1

\begin{tabular}{|c|c|c|}
\hline ply number & Snota & Thars: \\
\hline 1 & $0 \%$ & 0.083 \\
\hline 2 & $90^{\circ}$ & 0.026 \\
\hline 3 & 45 & 0,025 \\
\hline 4 & 45 & 0.325 \\
\hline F.eigen value & & $x$ \\
\hline
\end{tabular}

Eigen Value

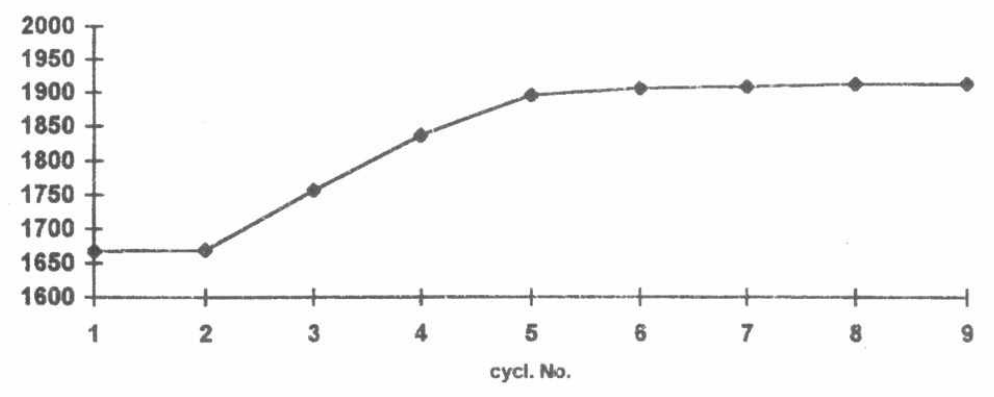

Fig. (1) History of frequency maximization 
Table 2 History of ply orientations in the frequency maximization level

\begin{tabular}{|c|c|c|c|c|c|c|c|c|c|}
\hline ryst. & 1 & 2. & 6. & 4 & 5 & 6. & 7 & 8 & 9 \\
\hline Ply & Angle & Angle & Angle & Angle & Angle & Angle & Angle & Angle & Angle \\
\hline 1 & 0 & -18.0 & -35.4 & -50 & -46.3 & -42.7 & -45.0 & -44 & -44.8 \\
\hline 2 & 89.7 & 75.0 & 57.3 & 25 & 25.9 & 29.3 & 31.0 & 33.6 & 45 \\
\hline 3 & 44.7 & 29.0 & 12.1 & 25 & 25.9 & 29.3 & 31.0 & 32.6 & 45 \\
\hline 4 & -45.2 & -60.9 & -64.6 & -50 & -46.3 & -42.7 & -45.0 & -44 & -44.8 \\
\hline Etgen & 1669 & 1756 & 1837 & 1897 & 1907.6 & 1910 & 1014 & $18 \times 5$ & 1921 \\
\hline
\end{tabular}

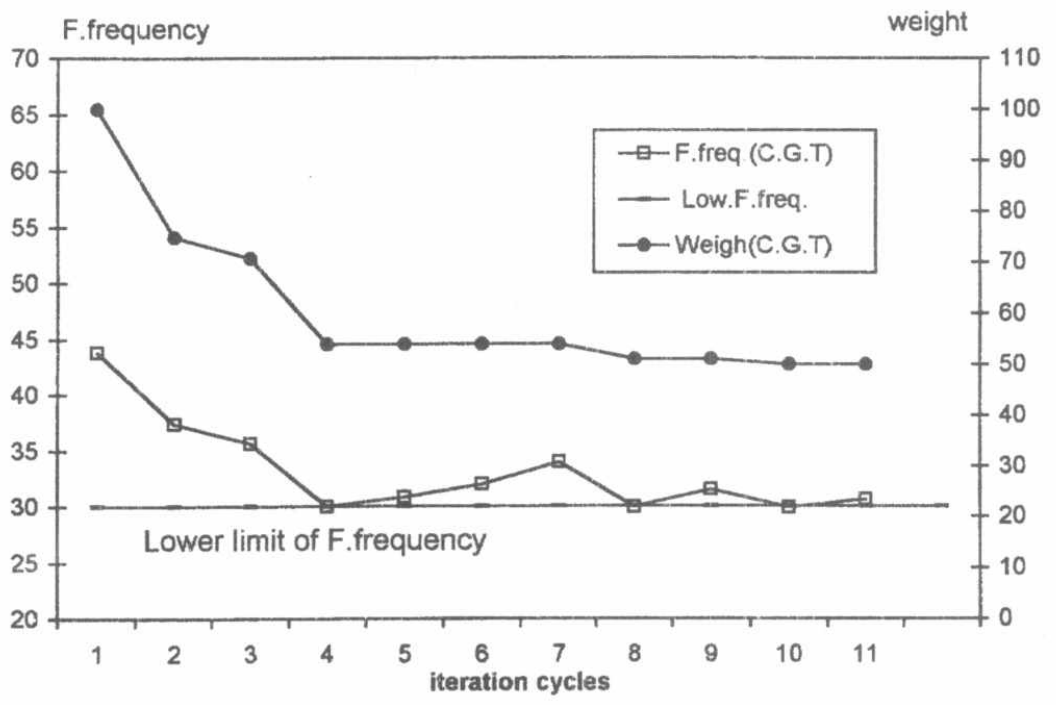

Fig. (2) History of weight minimization (C.G.T) 


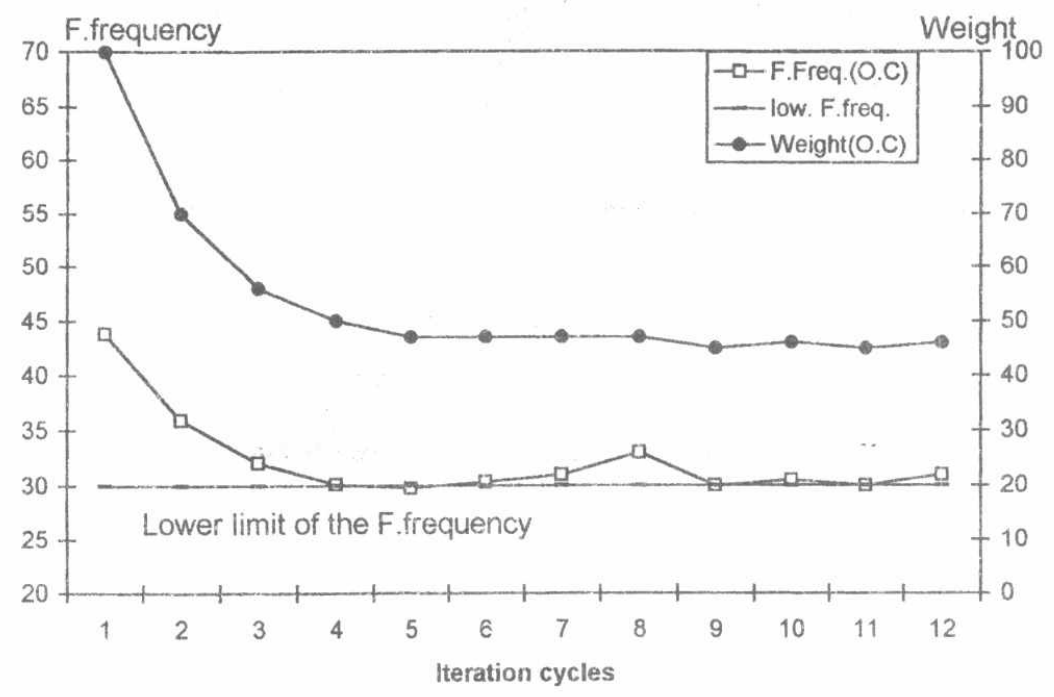

Fig. (3) History of weight minimization (O.C.)

\section{REFRENCES}

[1] Nasr A.N. and Negm H.M. , "Two New Simple Finite Elements for Static and Dynamic Analysis of Composite Plates ", J. of Engineering Appl. Science Faculty of engineering ,Cairo University, Vol.45,No.5, Pp.741-757 Oct(1998)

[2] Fleury C. and Braibant V.," Structural Optimization: A New Dual method Using Mixed Variables ",Int.j. Numer.Methods Eng.Vol.23,409-428(1986).

[3] Kiusalaas J and Shaw J. "An Algorithm for Optimal Structural Design with Frequency Constraints ", Int.J.Numer.Methods Eng.Vol.13,Pp.283-295 (1978).

[4] Rubin C. P.," Minimum Weight Design of Complex Structures Subject to a Frequency Constraint" AIAA Journal, Vol.8, No.5, May (1970).

[5] Schmit, L.A., " Structural Design by Systematic Synthesis", Proc. $2^{\text {nd }}$ Nat. Conf. Electronic computation, ASCE, pp.105-132(1960).

[6] Schmit L.A. and Fresh B.," Optimum Laminate Design for Strength and Stiffness ", Int.J.for Num.Meth.in Eng. Vol.9, Pp.519-531, (1973).

[7] Sobieszczanski-Sobiesk,J." Structural Sizing by Generalized, Mulilevel Optimization", AIAA j,Vol.25, NO.1,(1987).

[8] Vanderplaats G.N.," An Efficient Feasible Direction Algorithm for Design Synthesis.", AIAA Journal ,22,PP.1633-1640 (1984).

[9] Venkayya, V.B., "Design of Optimum Structures", Com\&Strut. 1,265-309 (1971).

[10] Zarghamee M.S., "Optimum Frequency Of Structures" AIAA Journal, Vol., No.4, PP.749-750, April (1968). 